in vivo $33: 2265-2272(2019)$

doi:10.21873/invivo.11732

\title{
Level and Value of $T$ Cell-derived Circulating Microparticles in Liver Cirrhosis Patients
}

\author{
CHIH-HUNG CHEN ${ }^{1}$, CHIA-LO CHANG ${ }^{2}$, KUAN-HUNG CHEN ${ }^{3}$, BEN-CHUNG CHENG ${ }^{4}$, \\ HONG-HWA CHEN ${ }^{2}$, JOHN Y. CHIANG ${ }^{5,6}$, PEI-HSUN SUNG ${ }^{7,8}$ and HON-KAN YIP IP $^{7,8,9,10,11}$ \\ ${ }^{1}$ Division of General Medicine, Department of Internal Medicine, Kaohsiung Chang Gung \\ Memorial Hospital and Chang Gung University College of Medicine, Kaohsiung, Taiwan, R.O.C.; \\ ${ }^{2}$ Division of Colorectal Surgery, Department of Surgery, Kaohsiung Chang Gung Memorial \\ Hospital and Chang Gung University College of Medicine, Kaohsiung, Taiwan, R.O.C.; \\ ${ }^{3}$ Department of Anesthesiology, Kaohsiung Chang Gung Memorial Hospital and \\ Chang Gung University College of Medicine, Kaohsiung, Taiwan, R.O.C.; \\ ${ }^{4}$ Division of Nephrology, Department of Internal Medicine, Kaohsiung Chang Gung Memorial \\ Hospital and Chang Gung University College of Medicine, Kaohsiung, Taiwan, R.O.C.; \\ ${ }^{5}$ Department of Computer Science and Engineering, National Sun Yat-Sen University, Kaohsiung, Taiwan, R.O.C.; \\ ${ }^{6}$ Department of Healthcare Administration and Medical Informatics, \\ Kaohsiung Medical University, Kaohsiung, Taiwan, R.O.C.; \\ ${ }^{7}$ Division of Cardiology, Department of Internal Medicine, Kaohsiung Chang Gung Memorial \\ Hospital and Chang Gung University College of Medicine, Kaohsiung, Taiwan, R.O.C.; \\ ${ }^{8}$ Center for Shockwave Medicine and Tissue Engineering, \\ Kaohsiung Chang Gung Memorial Hospital, Kaohsiung, Taiwan, R.O.C.; \\ ${ }^{9}$ Institute for Translational Research in Biomedicine, \\ Kaohsiung Chang Gung Memorial Hospital, Kaohsiung, Taiwan, R.O.C.; \\ ${ }^{10}$ Department of Medical Research, China Medical University Hospital, \\ China Medical University, Taichung, Taiwan, R.O.C.; \\ ${ }^{11}$ Department of Nursing, Asia University, Taichung, Taiwan, R.O.C.
}

\begin{abstract}
Background/Aim: We examined the hypothesis that $T$ cell-derived-circulating microparticles (MPs) are increased in liver-cirrhosis $(L C)$ patients compared to normal subjects and are also increased in chronic hepatitis compared to acutedecompensated-liver cirrhosis (ADLC). Patients and Methods: A total of 66 LC patients, including 35 with ADLC and 31 with non-decompensated-LC (NDLC), were enrolled in the study. Ten volunteers served as controls. Results: Flow-cytometric analysis showed that circulating levels of T-cell derived MPs (i.e., total
\end{abstract}

This article is freely accessible online.

Correspondence to: Pei-Hsun Sung and Hon-Kan Yip, Division of Cardiology, Department of Internal Medicine, Kaohsiung Chang Gung Memorial Hospital and Chang Gung University College of Medicine, 123, Dapi Road, Niaosung Dist., Kaohsiung city, 83301, Taiwan, R.O.C. Tel: +886 77317123, Fax: +886 77322402, e-mail: e12281@cgmh.org.tw; e-mail: han.gung@msa.hinet.net

Key Words: Circulating T-cell derived microparticles, inflammation, liver cirrhosis.
MPs and CD4+/CD8+/CD54+MPs) were higher in LC patients than in the controls (all $p<0.003$ ). Total MPs and CD8+MPs were higher in NDLC than in ADLC patients. There were good correlations between CD8+MPs and ADLC as well as between total MPs and chronic hepatitis. Multivariate-linear-regression analysis showed that NDLC was independently predictive of increased circulating CD + MPs levels $(p<0.05)$ and chronic hepatitis independently predictive of increased circulating total MPs levels $(p<0.001) / C D 4+M P s \quad(p<0.05)$. Conclusion: Circulating levels of T-cell-derived MPs were increased in ADLC patients and were even more elevated in NDLC patients compared to healthy-control subjects.

Liver cirrhosis (LC), a reversible wound-healing response to either acute or chronic cellular injury that reflects a balance between liver repair and scar formation (1), is the final pathological pathway of liver damage arising from a wide variety of chronic liver diseases (2-4). Clinical observational and epidemiologic studies have previously shown that LC is an increasing cause of morbidity and mortality in developed countries, being the 12th most 
common cause of death worldwide but fourth in central Europe (5-7).

It is well known that liver fibrosis/LC is a common pathological consequence of a variety of chronic stimuli, including viral infection, autoimmune, drug-induced, cholestatic and metabolic diseases (8-11). Studies have further clarified that the etiology of cirrhosis varies geographically, with alcoholism, chronic hepatitis $\mathrm{C}$ virus infection, and nonalcoholic fatty liver disease being the most common causes in western countries (12-14), whereas chronic hepatitis B is the primary cause of LC in the Asia-Pacific region (15-17).

Although the causes of LC are multifactorial, there are some pathological characteristics that are common to all cases of liver cirrhosis, including degeneration and necrosis of hepatocytes, replacement of liver parenchyma by fibrotic tissues and regenerative nodules, and loss of liver function (18-22). Additionally, a key discovery in understanding LC is initiated by activation of hepatic stellate cells (HSC), which are the primary effector cells orchestrating the deposition of extracellular matrix (ECM) in the liver structure. Furthermore, progressive accumulation and decreased remodeling of the ECM, disrupts the normal architecture of the liver (10). If left untreated, fibrosis can progress to LC, ultimately leading to organ failure and death (23).

Limited studies have previously shown that lymphocytes have the capacity to modulate fibroblast fibrolytic effects through lymphocyte-derived microparticles (MPs) rather by cytokine mediated manner $(24,25)$. Interestingly, one of these studies has further exhibited that T cell MPs circulated in blood and were elevated in patients with active chronic hepatitis $\mathrm{C}$ (25). Moreover, MPs derived both from CD8+ and CD4+ T cells can induce a fibrolytic phenotype in HSCs (25). These findings raise the hypothesis that circulating T-cell derived MPs may be a novel diagnostic marker for inflammatory liver diseases/LC (25). However, the available data to support this hypothesis is still largely limited, suggesting more prospective clinical studies are required to strengthen this issue.

Clinical significance. This study tested the hypothesis that circulating T-cell derived microparticles (MPs) might be a novel diagnostic marker for inflammatory liver diseases/liver cirrhosis (LC). Consistent to the hypothesis, the results of our study showed that circulating levels of T-cell derived MPs, indicators of inflammatory reaction in chronic hepatitis, were significantly increased in acute decompensated liver cirrhosis patients and more significantly increased in nondecompensated LC patients than in healthy-control subjects, highlighting a persistent inflammation in LC patients.

\section{Patients and Methods}

Study design. This clinical study was approved by the Institutional Review Committee on Human Research in Chang Gung Memorial Hospital (100-3666A3) in 2013 and conducted at Kaohsiung Chang
Gung Memorial Hospital. Informed consent was obtained from all study subjects.

Patient population, inclusion and exclusion criteria. Patients with age $>18$ years old who had a history of LC were enrolled into this prospective study. Patients with history of the followings were excluded from the study: intracranial hemorrhage, surgery or trauma within the preceding 3 months, hematology disorders, chronic renal disease in stage 5, malignancy other than hepatoma, febrile disorders, congestive heart failure, myeloproliferative disorder, without diagnosis of LC, acute or chronic inflammatory disease other than LC during the study period, history of autoimmune diseases with or without immunosuppressive therapy, and prior myocardial infraction with an onset of $<3$ months.

Between October 2013 and November 2014, 66 patients who presented to the Department of Gastroenterology at Kaohsiung Chang Memorial Hospital with the diagnosis of LC were recruited. Patients were enrolled either from the outpatient department or during hospitalization for severe jaundice, ascites with hypoalbuminemia for plasma transfusion, acute aspartate aminotransferase (AST) and alanine aminotransferase (ALT) elevation, esophageal varices ligation or esophageal varices bleeding (Figure 1). All blood samples were collected prior to blood transfusion.

Ten age- and gender-matched healthy controls were also studied. Informed consent was obtained from all healthy subjects.

For analytical purposes, the patients were categorized into acute decompensated liver cirrhosis (ADLC) (defined as Child's class B or C) and non-decompensated liver cirrhosis (NDLC) (defined as Child's class A).

Ultrasonographic examination and definitions. Ultrasonographic scans were performed by experienced hepatologists at our institute using an ultrasound system with a $3.5 \mathrm{MHz}$ convex probe (SSA340, SSA-370, Aplioxu-700 Toshiba, Tokyo, Japan; SSD 2000 , Aloka, Tokyo, Japan; HDI 5000, ATL Ultrasound, Bothell, WA, USA; Preirus Hi-vision Hitachi, Tokyo, Japan).

Chronic hepatitis $\mathrm{C}$ was defined as detectable serum antibody to hepatitis $\mathrm{C}$ virus (anti-HCV), and chronic hepatitis B was defined as detectable serum hepatitis B viral surface antigen (HBsAg). The definitions and diagnoses of hepatocellular carcinoma (HCC), LC, and the degree of decompensation were based on previous reports (26-30).

Circulating MPs were categorized into six groups. Circulating MPs were categorized into six types 1) Total MPs; (2) CD4+ apoptotic $\mathrm{T}$ cell (i.e., Annexin-V) derived MPs (i.e., CD4+ MPs); 3 ) CD8+ apoptotic T derived MPs (i.e., CD8+ MPs); 4) CD11a+ activated T cell (i.e., T-cell activating signal) (i.e., CD11a+ MPs); 5) CD54 (Cluster of differentiation 54), also known as ICAM-1 (intercellular adhesion molecule 1) is a member of the immunoglobulin superfamily (including antibodies and T-cell receptors) (i.e., CD54+ MPs); and 6) CD147 (also called basigin or EMMPRIN, is a transmembrane glycoprotein that belongs to the immunoglobulin superfamily) (i.e., CD147+ MPs). The procedure and protocol for classification have been outlined in a previous report (25) and used with minimal modification.

Blood samples for biochemical analysis, blood cell count study, and flow cytometric analysis of plasma levels of MPs. Blood samples were obtained once at 9:00 am and analysed according to our recent 




Figure 1. Flow chart illustrating the protocol of enrollment and allocation of study patients. ADLC: Acute decompensated liver cirrhosis; NDLC: non-decompensated liver cirrhosis.

reports $(31,32)$. In detail, white blood cell $(\mathrm{WBC})$ red blood cell (RB) and platelet counts, hemoglobin, biochemistry, and electrolyte levels were analyzed using standard laboratory methods in our hospital. Peripheral blood was collected from each study subject in acid citrate dextrose (ACD) vacutainer tubes. To prepare blood samples, peripheral blood $(1.5 \mathrm{ml})$ was centrifuged at $2000 \times g$ at $25^{\circ} \mathrm{C}$ for $20 \mathrm{~min}$ without acceleration or break. The $650 \mu \mathrm{l}$ plasma samples were transferred to a new Eppendorf and centrifuged for 2 $\min$ at $13,000 \times g$ at $25^{\circ} \mathrm{C}$ without break. The plasma samples were transfered to a new Eppendorf and centrifuged for $20 \mathrm{~min}$ at 13,000 $\times g$ at $25^{\circ} \mathrm{C}$ without break and then the pellet was collected for investigation of MPs smaller than $1.0 \mu \mathrm{m}$. Size calibration was conducted with $1.0 \mu \mathrm{m}$ beads (Invitrogen, Carlsbad, CA, USA). The MP pellet was resuspended in $150 \mu$ of Annexin- $V$ binding buffer (BD Biosciences). All buffers were sterile-filtered through a $0.2 \mu \mathrm{m}$ filter. The $100 \mu \mathrm{l}$ MPs were then incubated in a TruCOUNT tube (BD Biosciences) with fluorescent monoclonal antibodies: 1) Annexin V-FITC; 2) CD4-PE; 3) CD8-PE; 4) CD11a-PE; 5) CD54$\mathrm{PE}$; and 6) CD147-PE (BD Biosciences). The samples were incubated in the dark for $15 \mathrm{~min}$ at room temperature. The samples were then analyzed on a FC500 flow cytometer (Beckman Coulter) after the addition of $400 \mu \mathrm{l}$ Annexin-V binding buffer. The absolute count of MPs was measured setting the stop condition for TruCount beads at 10,000 events.

Statistical analysis. Continuous variables with normal distribution were expressed as mean \pm standard deviation, and the difference between the two groups was analyzed with independent t-test. We compared continuous variables among the three groups using oneway analysis of variance with post-hoc Bonferroni test. Discrete or categorical variables between different groups were reported as percentage and number, and then analyzed with Chi-square test or Fisher's exact test as appropriate. Additionally, Pearson's correlation analysis was adopted to assess the relation between the level of MPs and disease status. Furthermore, after logarithmization of MPs, the multivariate linear regression model was utilized to identify clinical or pathological predictors of circulating MPs change. Statistical analysis was performed using SPSS statistical software for Windows version 22 (SPSS for Windows, version 22; SPSS, IL, USA). $p<0.05$ was considered as statistically significant.

\section{Results}

Comparisons of baseline characteristics of study patients and control subjects (Table I). Table I shows the baseline characteristics of study patients and healthy volunteers. The results demonstrated that age, gender, body weight, body height, RBC count, WBC count, hemoglobin, circulating levels of AST, ALT, alkaline-phosphatase and creatinine did not differ between study patients and control subjects. However, the platelet count was significantly lower whereas the total number of the MPs were significantly higher in patients compared to control subjects. Additionally, circulating levels of MPs, including CD4+, CD8+ and CD54+ MPs were significantly increased in patients compared to control subjects.

Comparison of baseline characteristics between patients with acute decompensated liver cirrhosis (ADLC) and non- 
in vivo $33: 2265-2272(2019)$

Table I. Comparison of baseline characteristics between symptomatic liver cirrhotic $(L C)$ patients and control subjects.

\begin{tabular}{lccc}
\hline Variables & Control group $(\mathrm{n}=10)$ & Study group $(\mathrm{n}=66)$ & $p$-Value \\
\hline Age $(\mathrm{yrs})$ & $49.70 \pm 8.00$ & $56.76 \pm 13.77$ & 0.120 \\
Male gender & $50 \%(5)$ & $65.2 \%(43)$ & 0.484 \\
Body weight $(\mathrm{kg})$ & $63.20 \pm 8.23$ & $63.77 \pm 12.72$ & 0.915 \\
Body height $(\mathrm{cm})$ & $162.14 \pm 5.84$ & $163.28 \pm 8.35$ & 0.730 \\
WBC count $\left(\times 10^{3} / \mathrm{ml}\right)$ & $8.21 \pm 2.26$ & $6.92 \pm 3.14$ & 0.294 \\
RBC count $\left(\times 10^{6} / \mathrm{ml}\right)$ & $5.56 \pm 2.37$ & $4.06 \pm 0.93$ & 0.147 \\
Platelet count $\left(\times 10^{3} / \mathrm{ml}\right)$ & $248.43 \pm 46.22$ & $150.58 \pm 71.90$ & $0.001^{*}$ \\
Hemoglobin $(\mathrm{g} / \mathrm{dl})$ & $17.01 \pm 7.34$ & $12.58 \pm 2.38$ & 0.162 \\
AST $(\mathrm{IU} / \mathrm{l})$ & $27.67 \pm 8.14$ & $260.45 \pm 538.57$ & 0.297 \\
ALT $(\mathrm{IU} / \mathrm{l})$ & $27.86 \pm 18.46$ & $309.71 \pm 742.71$ & 0.322 \\
Alkaline-Phosphatase $(\mathrm{IU} / \mathrm{l})$ & $61.6 \pm 18.46$ & $124.17 \pm 103.12$ & 0.185 \\
Creatinine level $(\mathrm{mg} / \mathrm{dl})$ & $0.79 \pm 0.27$ & $0.88 \pm 0.32$ & 0.449 \\
Total microparticles $(\mathrm{MPs} / \mathrm{ml}) \times 10^{6}$ & $0.23 \pm 0.21$ & $2.03 \pm 3.47$ & $<0.001^{*}$ \\
CD4+ $(\mathrm{MPs} / \mathrm{ml}) \times 10^{6}$ & $0.12 \pm 0.06$ & $0.43 \pm 0.5$ & $<0.001^{*}$ \\
CD8 $(\mathrm{MPs} / \mathrm{ml}) \times 10^{6}$ & $0.11 \pm 0.07$ & $0.55 \pm 0.74$ & $<0.001^{*}$ \\
CD11a+ $(\mathrm{MPs} / \mathrm{ml}) \times 10^{6}$ & $1.00 \pm 0.67$ & $2.14 \pm 2.95$ & 0.228 \\
CD54+ $(\mathrm{MPs} / \mathrm{ml}) \times 10^{6}$ & $0.20 \pm 0.16$ & $0.47 \pm 0.56$ & $0.002^{*}$ \\
CD147+ $(\mathrm{MPs} / \mathrm{ml}) \times 10^{6}$ & $3.59 \pm 2.55$ & $6.80 \pm 7.54$ & 0.118 \\
\hline
\end{tabular}

Independent $t$-test; Chi-Square test (+/- Fisher exact test). WBC: White blood cell; RBC: red blood cell; AST: aspartate aminotransferase; ALT: alanine aminotransferase; MPs: microparticles.

decompensated liver cirrhosis (NDLC) (Table II). Age, gender, body weight, body height, WBC and platelet counts did not differ between the two groups of patients. However, the RBC count and hemoglobin levels were significantly lower in the ADLC group than those in the NDLC group.

The circulating levels of creatinine, AST, ALT, total bilirubin, $\alpha$-fetal protein and ammonia were similar between these two groups of the patients. Additionally, the incidences of hepatitis B carrier, hepatitis C carrier and hepatocellular carcinoma did not differ between these two groups of patients. However, the incidences of alcoholic LC, splenomegaly and esophageal varices bleeding were significantly higher whereas acute hepatis was significant lower in the ADLC group than in the NDLC group.

Correlation between ADLC and microparticles (Table III). The Pearson's correlation analysis was utilized to examine the correlation between the ADCL and circulating level of MPs. The results showed that only CD8+ MPs were significantly correlated with the ADLC. On the other hand, the circulating levels of CD4+ MPs had a tendency of significant correlation with ADLC.

Correlation between chronic hepatitis and microparticles (Table IV). The Pearson's correlation analysis was also utilized to examine the correlation between chronic hepatitis and circulating levels of MPs. The results showed that only total MPs had a strong significant correlation with chronic hepatitis.
Multivariate linear regression analysis of the predictors of increased circulating microparticles (Table V). The analytical results showed that the change in circulating CD8+ MPs was significantly predictive of NDLC. Additionally, circulating levels of total MPs and CD4+ MPs were significantly influenced by the existence of chronic hepatitis.

Mean levels of total and different microparticles in control subjects, ADLC and NDLC (Figures 2 and 3). Figures 2 and 3 show that the levels of total MPs and different kinds of MPs (i.e., CD4+, CD8+, CD11a+ MPs, CD54+ and CD147+) were notably higher in ADLC patients and more notably higher in NDLC patients than in control subjects.

\section{Discussion}

This study investigated the circulating levels of MPs in LC patients and yielded several striking clinical implications. First, as compared with healthy control subjects, the circulating levels of total MPs and different kinds of MPs (i.e., CD4+, CD8+, CD54+) were substantially higher in ADLC and more substantially higher in NDLC patients, highlighting that presence of persistent inflammation in LC patients. Second, the results of multivariate linear regression analysis showed that NDLC rather than ADLC was significantly predictive of increasing circulating levels of MPs (i.e., CD8+MPs), suggesting that the inflammatory response was vigorous in the former than in the later situation. Third, the results showed that chronic hepatitis was 
Table II. Comparison of baseline characteristics between patients with acute decompensated liver cirrhosis (ADLC) and non-decompensated liver cirrhosis (NDLC).

\begin{tabular}{lccc}
\hline Variables & ADLC group $(\mathrm{n}=35)$ & NDLC group $(\mathrm{n}=31)$ & $p$-Value \\
\hline Age $(\mathrm{yrs})$ & $56.94 \pm 12.67$ & $56.55 \pm 15.13$ & 0.910 \\
Male gender & $68.6 \%(24)$ & $61.3 \%(19)$ & 0.536 \\
Body weight $(\mathrm{kg})$ & $63.30 \pm 13.72$ & $64.44 \pm 11.48$ & 0.769 \\
Body height $(\mathrm{cm})$ & $163.93 \pm 8.47$ & $162.37 \pm 8.33$ & 0.538 \\
WBC count $\left(\times 10^{3} / \mathrm{ml}\right)$ & $6.83 \pm 3.04$ & $7.03 \pm 3.33$ & 0.808 \\
RBC count $\left(\times 10^{6} / \mathrm{ml}\right)$ & $3.79 \pm 0.88$ & $4.42 \pm 0.88$ & 0.008 \\
Platelet count $\left(\times 10^{3} / \mathrm{ml}\right)$ & $145.38 \pm 81.51$ & $157.38 \pm 57.86$ & 0.526 \\
Hemoglobin $(\mathrm{g} / \mathrm{dl})$ & $11.99 \pm 2.50$ & $13.34 \pm 2.01$ & 0.028 \\
AST $(\mathrm{IU} / \mathrm{l})$ & $151.63 \pm 231.0$ & $394.38 \pm 749.23$ & 0.122 \\
ALT $(\mathrm{IU} / \mathrm{l})$ & $128.12 \pm 314.18$ & $522.62 \pm 1009.01$ & 0.051 \\
Alkaline-phosphatase $(\mathrm{IU} / \mathrm{l})$ & $151.87 \pm 133.49$ & $98.68 \pm 55.45$ & 0.086 \\
Creatinine level $(\mathrm{mg} / \mathrm{dl})$ & $0.88 \pm 0.33$ & $0.87 \pm 0.31$ & 0.903 \\
Total bilirubin & $3.21 \pm 4.70$ & $2.67 \pm 5.22$ & 0.676 \\
$\alpha$-fetal protein $(\mu \mathrm{g} / \mathrm{l})$ & $3350.05 \pm 16326.43$ & $67.31 \pm 273.19$ & 0.335 \\
Ammonia & $95.59 \pm 58.73$ & $77.43 \pm 30.72$ & 0.287 \\
Splenomegaly & $58.8 \%(20)$ & $23.1 \%(6)$ & 0.006 \\
Hepatitis B carrier & $17.1 \%(6)$ & $22.6 \%(7)$ & 0.579 \\
Hepatitis C carrier & $22.9 \%(8)$ & $22.6 \%(7)$ & 0.979 \\
Acute hepatitis & $2.9 \%(1)$ & $25.8 \%(8)$ & 0.010 \\
Alcoholic liver cirrhosis & $37.1 \%(13)$ & $0.0 \%(0)$ & $<0.001$ \\
Hepatocellular carcinoma & $11.4 \%(4)$ & $9.7 \%(3)$ & 1.000 \\
EV bleeding & $59.3 \%(16)$ & $18.2 \%(2)$ & 0.033 \\
\hline
\end{tabular}

Independent $t$-test or Chi-Square test (+/- Fisher exact test) were utilized. WBC: White blood cell; RBC: red blood cell; AST: aspartate aminotransferase; ALT: alanine aminotransferase; EV: esophageal varices.

Table III. Correlation between the ADLC and microparticles.

\begin{tabular}{lcccccc}
\hline Variables & Total MPs & CD4+ MPs & CD8+ MPs & CD11a+ MPs & CD54+ MPs & CD147+ MPs \\
\hline $\mathrm{r}$ & 0.169 & 0.212 & 0.262 & 0.176 & 0.138 & 0.160 \\
$p$-Value & 0.144 & 0.066 & $0.022^{*}$ & 0.128 & 0.234 & 0.174 \\
\hline
\end{tabular}

ADLC: Acute decompensated liver cirrhosis; MP: microparticles.

Table IV. Correlation between the chronic hepatitis* and microparticles.

\begin{tabular}{lcccccc}
\hline Variables & Total MPs & CD4+ MPs & CD8+ MPs & CD11a+ MPs & CD54+ MPs & CD147+ MPs \\
\hline $\mathrm{r}$ & 0.390 & 0.211 & 0.211 & 0.167 & 0.175 & 0.192 \\
$p$-Value & $<0.001$ & 0.068 & 0.423 & 0.150 & 0.131 & 0.102 \\
\hline
\end{tabular}

MPs: Microparticles. *Chronic hepatitis: defined as hepatitis B and C carriers.

the strongest independent predictor of increased circulating levels of total MPs and CD4+ MPs.

Currently, there are still limited data to address the correlation between hepatitis and increased levels of $\mathrm{T}$ cell derived circulating MPs (25). To the best of our knowledge, this is the second reported study to address human $\mathrm{T}$ cell derived MPs circulating in blood of hepatitis/LC patients. Accordingly, our findings would provide an additional information for the readers to understand the role of $\mathrm{T}$ cell derived MPs in the process of chronic hepatitis and LC.

One important finding of the present study was that, compared to healthy control subjects, the circulating levels 




Figure 2. Flow cytometric analysis of total circulating microparticles (MPs) among normal ( $n=10)$, ADLC ( $n=35)$, and NDLC ( $n=31)$ groups. The cirrhotic patients had a significant higher mean of total MPs than the normal population $(p<0.001)$, whereas there was no significant difference in total MPs between ADLC and NDLC groups $(p=0.253)$. Letters $(a, b)$ were used for comparison of the same parameter among different groups, indicating significant difference $(p<0.05)$ with one-way ANOVA. ADLC: Acute decompensated liver cirrhosis; NDLC: non-decompensated liver cirrhosis.

Table V. Multivariate linear regression analysis of predictors of increased circulating levels of microparticles (MPs).

\begin{tabular}{|c|c|c|c|c|c|c|}
\hline \multirow{2}{*}{$\begin{array}{l}\text { MPs } \\
\text { Variables }\end{array}$} & \multicolumn{2}{|c|}{ Total MPs } & \multicolumn{2}{|c|}{$\mathrm{CD} 4+\mathrm{MP}$} & \multicolumn{2}{|c|}{$\mathrm{CD} 8+\mathrm{MP}$} \\
\hline & Coeff. & $95 \% \mathrm{CI}$ & Coeff. & $95 \% \mathrm{CI}$ & Coeff. & $95 \% \mathrm{CI}$ \\
\hline ADLC & -0.028 & - & 0.007 & - & 0.269 & - \\
\hline NDLC & 0.073 & - & 0.038 & - & $0.676^{*}$ & $(0.01,1.35)$ \\
\hline Age & -0.042 & - & 0.069 & - & 0.065 & - \\
\hline Gender & 0.063 & - & -0.035 & - & 0.044 & - \\
\hline Chronic hepatitis & $1.439 * * *$ & $(0.66,2.22)$ & $0.714 *$ & $(0.07,1.36)$ & 0.053 & - \\
\hline Acute hepatitis & 0.169 & - & 0.004 & - & 0.106 & - \\
\hline Alcoholic LC & 0.027 & - & -0.007 & - & 0.176 & - \\
\hline $\mathrm{HCC}$ & 0.075 & - & 0.139 & - & -0.003 & - \\
\hline EV bleeding & 0.013 & - & 0.176 & - & 0.133 & - \\
\hline
\end{tabular}

Coeff.: Coefficient; CI: confidence interval; ADLC: acute decompensated liver cirrhosis; NDLC: non-decompensated liver cirrhosis; HCC: hepatocellular carcinoma; EV bleeding: esophageal variceal bleeding. Superscripts *, **, and *** indicate $p<0.05, p<0.01$, and $p<0.001$, respectively. Data of $95 \% \mathrm{CI}$ are expressed if statistically significant.

of MPs were remarkably increased in LC patients. A previous study (25) has shown that $\mathrm{T}$ cell derived MPs (i.e., CD4+, CD8+, CD54+, CD147) were strongly correlated with inflammatory liver diseases. Our findings, which are in agreement with this study (25), indicate that our LC patients were at a situation of inflammation that could result in chronic persistent hepatitis.

A previous study (25) has shown that MPs which circulate in blood could be a novel diagnostic marker for diagnosis of hepatic diseases and also represent a novel strategy to induce 


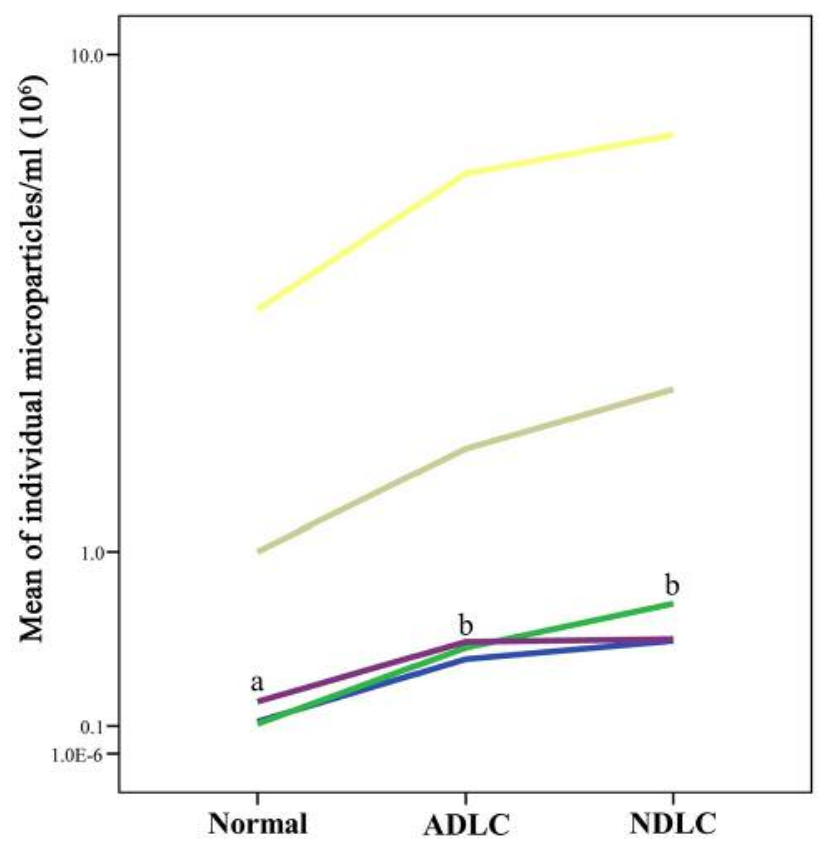

Type of MPs

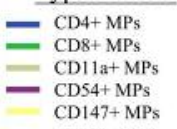

Figure 3. Flow cytometric analysis of individual circulating MPs among three groups. The mean values of circulating $C D 4+, C D 8+$ and $C D 54+$ $M P s$ were significantly higher in the cirrhotic than in the normal group (all p-values <0.002). However, the mean circulating MPs of CD11a+ and CD147+ didn't differ between the cirrhotic and normal group. Additionally, all five individual circulating MPs also had no significant difference between the ADLC and NDLC groups. Letters $(a, b)$ were used for comparison of the same parameter among different groups, indicating significant difference $(p<0.05)$ with one-way ANOVA. ADLC: Acute decompensated liver cirrhosis; NDLC: non-decompensated liver cirrhosis.

regression of LC. The most important finding in the present study was that the circulating levels of total MPs and CD4+ MPs were significantly independently predictive of chronic hepatitis. In this way, our findings were consistent with the finding of this previous study (25).

An essential finding of the present study was that a significant correlation was identified between ADLC and circulating levels of CD8+ MPs. However, the multivariate linear regression analysis, showed that only NDLC rather than ADLC was significantly independently predictive of increased circulating levels of MPs (i.e., CD8+MPs). Our finding indicated that a persistent and more rigorous inflammatory reaction might be activated in NDLC than in ADLC.

This study has limitations. First the sample size was relatively small and the standard deviation of circulating
MPs was extremely high which, therefore, would distort statistical significance. Second, LC was resulted from different disease entities. This might have resulted in unreliable Pearson's correlation coefficient " $R$ " and coefficient values.

\section{Conclusion}

In conclusion, circulating levels of T cell-derived MPs may be a useful accessory biomarker for predicting active inflammation in chronic hepatitis and LC.

\section{Conflicts of Interest}

The Authors report no conflicts of interest regarding this study.

\section{Authors' Contributions}

C.-H.C. and K.-H.C.: study design, data acquisition, analysis, drafting of manuscript; C.-H.C., and C.-L.C: laboratory assay and troubleshooting; B.-C.C., H.-H.C., and J.-Y.C: data acquisition, analysis, and interpretation; P.-H.S. and H.-K.Y.: study conception and design, coordination, drafting of manuscript.

\section{Acknowledgements}

This work was supported by research grants from Chang Gung Memorial Hospital, Chang Gung University (Grant no. CMRPG8C0471).

\section{References}

1 Lee UE and Friedman SL: Mechanisms of hepatic fibrogenesis. Best Pract Res Clin Gastroenterol 25(2): 195-206, 2011. PMID: 21497738. DOI: 10.1016/j.bpg.2011.02.005

2 Asrani SK, Larson JJ, Yawn B, Therneau TM and Kim WR: Underestimation of liver-related mortality in the united states. Gastroenterology 145(2): 375-382 e371-372, 2013. PMID: 23583430. DOI: $10.1053 /$ j.gastro.2013.04.005

3 Melato $M$ and Mucli E: Something new in liver cirrhosis epidemiology. Lancet 2(8659): 395-396, 1989. PMID: 2569588. DOI: $10.1016 / \mathrm{s} 0140-6736(89) 90578-3$

4 Qua CS and Goh KL: Liver cirrhosis in malaysia: Peculiar epidemiology in a multiracial asian country. J Gastroenterol Hepatol 26(8): 1333-1337, 2011. PMID: 21443669. DOI: 10.1111/j.1440-1746.2011.06732.x

5 D'Amico G, Garcia-Tsao G and Pagliaro L: Natural history and prognostic indicators of survival in cirrhosis: A systematic review of 118 studies. J Hepatol 44(1): 217-231, 2006. PMID: 16298014. DOI: $10.1016 /$ j.jhep.2005.10.013

6 Lau T and Ahmad J: Clinical applications of the model for endstage liver disease (meld) in hepatic medicine. Hepat Med 5: 110, 2013. PMID: 24696621. DOI: 10.2147/HMER.S9049

7 Moreau R, Jalan R, Gines P, Pavesi M, Angeli P, Cordoba J, Durand F, Gustot T, Saliba F, Domenicali M, Gerbes A, Wendon J, Alessandria C, Laleman W, Zeuzem S, Trebicka J, Bernardi M, Arroyo V and CANONIC Study Investigators of the EASL-CLIF 
Consortium: Acute-on-chronic liver failure is a distinct syndrome that develops in patients with acute decompensation of cirrhosis. Gastroenterology 144(7): 1426-1437, 1437 e1421-1429, 2013. PMID: 23474284. DOI: 10.1053/j.gastro.2013.02.042

8 Cohen-Naftaly $M$ and Friedman SL: Current status of novel antifibrotic therapies in patients with chronic liver disease. Therap Adv Gastroenterol 4(6): 391-417, 2011. PMID: 22973420. DOI: $10.1177 / 1756283$ X11413002

9 Hernandez-Gea V and Friedman SL: Pathogenesis of liver fibrosis. Annu Rev Pathol 6: 425-456, 2011. PMID: 21073339. DOI: 10.1146/annurev-pathol-011110-130246

10 Iredale JP: Models of liver fibrosis: Exploring the dynamic nature of inflammation and repair in a solid organ. J Clin Invest 117(3): 539-548, 2007. PMID: 17332881 . DOI: $10.1172 /$ JCI30542

11 Puche JE, Saiman Y and Friedman SL: Hepatic stellate cells and liver fibrosis. Compr Physiol 3(4): 1473-1492, 2013. PMID: 25855820. DOI: $10.1002 /$ cphy.c120035

12 Di Bisceglie AM: Natural history of hepatitis c: Its impact on clinical management. Hepatology 31(4): 1014-1018, 2000. PMID: 10733560 . DOI: 10.1053/he.2000.5762

13 Innes HA, Hutchinson SJ, Barclay S, Cadzow E, Dillon JF, Fraser A, Goldberg DJ, Mills PR, McDonald SA, Morris J, Stanley A and Hayes P: Quantifying the fraction of cirrhosis attributable to alcohol among chronic hepatitis c virus patients: Implications for treatment cost-effectiveness. Hepatology 57(2): 451-460, 2013. PMID: 22961861. DOI: 10.1002/hep.26051

14 Naveau S, Perlemuter G and Balian A: Epidemiology and natural history of cirrhosis. Rev Prat 55(14): 1527-1532, 2005. PMID: 16255293.

15 Ganem D and Prince AM: Hepatitis b virus infection - natural history and clinical consequences. N Engl J Med 350(11): 11181129, 2004. PMID: 15014185. DOI: 10.1056/NEJMra031087

16 Liaw YF, Leung N, Kao JH, Piratvisuth T, Gane E, Han KH, Guan R, Lau GK, Locarnini S and Chronic Hepatitis B Guideline Working Party of the Asian-Pacific Association for the Study of the Liver: Asian-pacific consensus statement on the management of chronic hepatitis b: A 2008 update. Hepatol Int 2(3): 263-283, 2008. PMID: 26811110. DOI: 10.1007/s12072-008-9080-3

17 Lv GC, Yao JM, Yang YD, Zheng L, Sheng JF, Chen Y and Li LJ: Efficacy of combined therapy in patients with hepatitis $b$ virus-related decompensated cirrhosis. World J Gastroenterol 19(22): 3481-3486, 2013. PMID: 23801842. DOI: 10.3748/ wjg.v19.i22.3481

18 Anthony PP, Ishak KG, Nayak NC, Poulsen HE, Scheuer PJ and Sobin LH: The morphology of cirrhosis. Recommendations on definition, nomenclature, and classification by a working group sponsored by the world health organization. J Clin Pathol 31(5): 395-414, 1978. PMID: 28850992. DOI: 10.1136/jcp.31.5.395

19 Elsharkawy AM, Oakley F and Mann DA: The role and regulation of hepatic stellate cell apoptosis in reversal of liver fibrosis. Apoptosis 10(5): 927-939, 2005. PMID: 26811110. DOI: $10.1007 / \mathrm{s} 10495-005-1055-4$

20 Ferrell L: Liver pathology: Cirrhosis, hepatitis, and primary liver tumors. Update and diagnostic problems. Mod Pathol 13(6): 679704, 2000. PMID: 20700952. DOI: 10.1038/modpathol.3880119

21 Wanless IR, Nakashima E and Sherman M: Regression of human cirrhosis. Morphologic features and the genesis of incomplete septal cirrhosis. Arch Pathol Lab Med 124(11): 1599-1607, 2000. PMID: 11079009. DOI: 10.1043/0003-9985(2000)124<1599: $\mathrm{ROHC}>2.0 . \mathrm{CO} ; 2$
22 Zhou WC, Zhang QB and Qiao L: Pathogenesis of liver cirrhosis. World J Gastroenterol 20(23): 7312-7324, 2014. PMID: 24966602. DOI: 10.3748/wjg.v20.i23.7312

23 Elpek GO: Cellular and molecular mechanisms in the pathogenesis of liver fibrosis: An update. World J Gastroenterol 20(23): 72607276, 2014. PMID: 24966597. DOI: 10.3748/wjg.v20.i23.7260

24 Distler JH, Jungel A, Huber LC, Seemayer CA, Reich CF, 3rd, Gay RE, Michel BA, Fontana A, Gay S, Pisetsky DS and Distler $\mathrm{O}$ : The induction of matrix metalloproteinase and cytokine expression in synovial fibroblasts stimulated with immune cell microparticles. Proc Natl Acad Sci USA 102(8): 2892-2897, 2005. PMID: 15701693. DOI: 10.1073/pnas.0409781102

25 Kornek M, Popov Y, Libermann TA, Afdhal NH and Schuppan D: Human t cell microparticles circulate in blood of hepatitis patients and induce fibrolytic activation of hepatic stellate cells. Hepatology 53(1): 230-242, 2011. PMID: 25855820. DOI: 10.1002/hep.23999

26 Baik SK, Jee MG, Jeong PH, Kim JW, Ji SW, Kim HS, Lee DK, Kwon SO, Kim YJ, Park JW and Chang SJ: Relationship of hemodynamic indices and prognosis in patients with liver cirrhosis. Korean J Intern Med 19(3): 165-170, 2004. PMID: 15481608. DOI: $10.3904 / \mathrm{kjim} .2004 .19 .3 .165$

27 Hung CH, Lu SN, Wang JH, Lee CM, Chen TM, Tung HD, Chen $\mathrm{CH}$, Huang WS and Changchien CS: Correlation between ultrasonographic and pathologic diagnoses of hepatitis $\mathrm{b}$ and $\mathrm{c}$ virus-related cirrhosis. J Gastroenterol 38(2): 153-157, 2003. PMID: 26811110. DOI: 10.1007/s005350300025

28 Kim MY, Baik SK, Yea CJ, Lee IY, Kim HJ, Park KW, Kim HK, Suk KT, Kim JW, Kim HS, Kwon SO, Cha SH, Kim YJ, Koh SB and Chang SJ: Hepatic venous pressure gradient can predict the development of hepatocellular carcinoma and hyponatremia in decompensated alcoholic cirrhosis. Eur J Gastroenterol Hepatol 21(11): 1241-1246, 2009. PMID: 19455045. DOI: 10.1097/MEG.0b013e32832a21c1

29 Pugh RN, Murray-Lyon IM, Dawson JL, Pietroni MC and Williams R: Transection of the oesophagus for bleeding oesophageal varices. Br J Surg 60(8): 646-649, 1973. PMID: 25855820. DOI: $10.1002 /$ bjs. 1800600817

30 Wang JH, Changchien CS, Hung CH, Eng HL, Tung WC, Kee $\mathrm{KM}$, Chen $\mathrm{CH}, \mathrm{Hu} \mathrm{TH}$, Lee $\mathrm{CM}$ and $\mathrm{Lu} \mathrm{SN}$ : Fibroscan and ultrasonography in the prediction of hepatic fibrosis in patients with chronic viral hepatitis. J Gastroenterol 44(5): 439-446, 2009. PMID: 19308312. DOI: 10.1007/s00535-009-0017-y

31 Tseng CC, Wang CC, Chang HC, Tsai TH, Chang LT, Huang $\mathrm{KT}$, Leu S, Yen $\mathrm{CH}$, Liu SF, Chen $\mathrm{CH}$, Yang CT, Yip HK and Lin MC: Levels of circulating microparticles in lung cancer patients and possible prognostic value. Dis Markers 35(5): 301310, 2013. PMID: 24167378. DOI: $10.1155 / 2013 / 715472$

32 Wang CC, Tseng CC, Hsiao CC, Chang HC, Chang LT, Fang WF, Leu S, Wang YH, Tsai TH, Yang CT, Chen CH, Yip HK, Ho CK and Lin MC: Circulating endothelial-derived activated microparticle: A useful biomarker for predicting one-year mortality in patients with advanced non-small cell lung cancer. Biomed Res Int 2014: 173401, 2014. PMID: 25061601. DOI: $10.1155 / 2014 / 173401$ 